\title{
A NOVEL APPROACH TO SCIATIC NERVE BLOCKADE (A CAN'T GA, CAN'T SPINAL SCENARIO)
}

C McGarrigle, G Gormley, J McDonnell

Grúpa Ollscoile Cúram Släinte

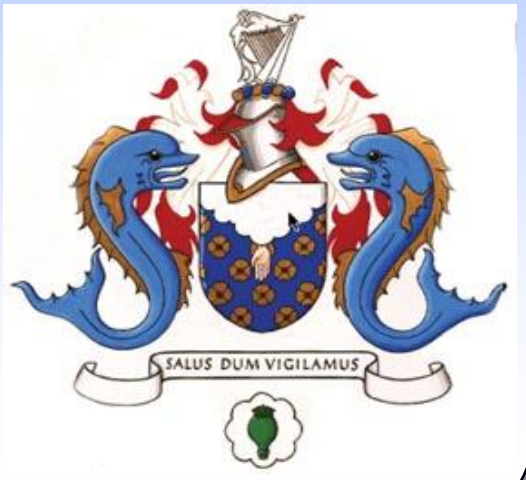

\section{Introduction/Background and Aims}

An elderly polytrauma patient in extremis, requiring emergency lower limb surgery, unsuitable for general anaesthesia or central neuraxial blockade due to extensive lung injuries and vertebral fractures. The patient's positioning was dictated by his injuries and he remained supine throughout.

\section{Methods}

A combined femoral sciatic nerve block was completed. An anterior approach to the sciatic nerve block was employed. Ultrasound guidance using longitudinal views over conventional transverse views were used, as visual optimisation of the sciatic nerve from leg rotation was not possible due to the patient's inferior pubic rami fracture.
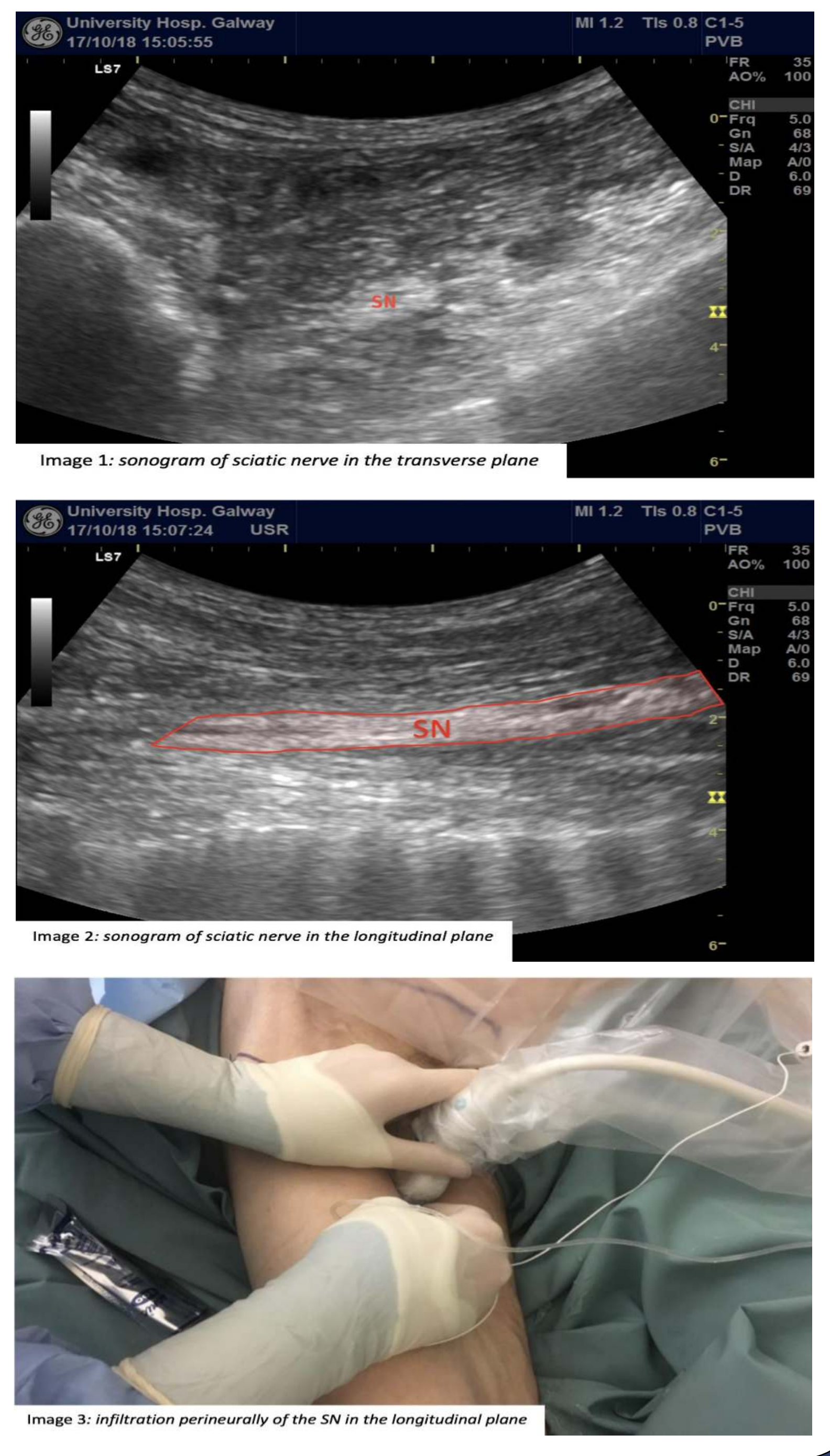

\section{Results}

Visualisation of the SN was achieved through longitudinal ultrasound views. Longitudinal views allowed the SN to be viewed as a distinctly continuous hyperechoic structure with a characteristic fascicular pattern, differentiating it from its surrounding muscle layers. Longitudinal views allowed an in-plane technique to be utilised. Accurate visualisation of the direct spread of local anaesthetic around the sciatic nerve was achieved, ensuring optimal blockade. The patient remained comfortable and haemodynamically stable throughout the surgery.
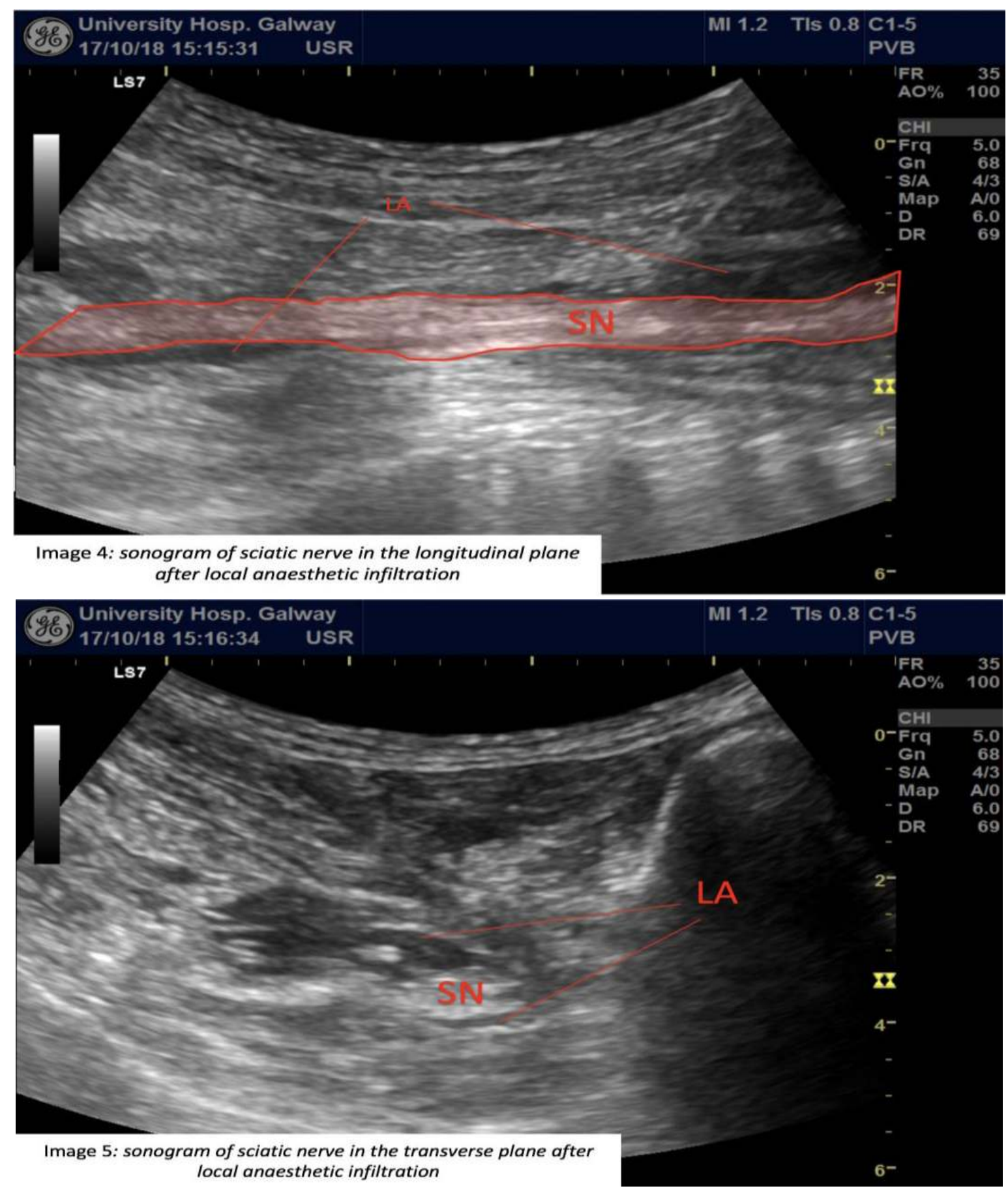

\section{Conclusions}

A combined femoral sciatic nerve block proved a safe and effective alternative for lower extremity anaesthesia. An anterior approach to the sciatic nerve block was performed, due to the necessity of remaining in a supine position. Unconventional longitudinal views facilitated the observation of the sciatic nerve and subsequent spread of local anaesthetic, while allowing an in-plane technique, thus ensuring optimal blockade.

\section{References}

Ota J, Sakura S, Hara K, Saito Y. Ultrasound-guided anterior approach to sciatic nerve block: a comparison with the posterior approach. Anesth Analg. 2009;108(2):660-5

Bansal L, Attri JP, Verma P. Lower limb surgeries under combined femoral and sciatic nerve block. Anesth Essays Res. 2016;10(3):432-436. doi:10.4103/0259-1162.177186

Vloka JD, Hadzic A, April E, Thys DM. Anterior approach to the sciatic nerve block: The effects of leg rotation. Anesth Analg. 2001;92:460-2.

Tsui BC, Ozelsel TJ. Ultrasound-guided anterior sciatic nerve block using a longitudinal approach: "expanding the view". Reg Anesth Pain Med. 2008;33:275-6. 